\title{
HEAVY FLAVOUR WEAK TRANSITIONS ON THE LATTICE
}

\author{
M.B. Gavela $a^{*}$ ) \\ Dept. de Fisica Teorica \\ Univ. Autonoma de Madrid \\ L. Maiani and S. Petrarca \\ Istituto di Fisica "G. Marconi" \\ Università degli Studi di Roma "La Sapienza" \\ and INFN, Sezione di Roma \\ G. Martine $11 i$ \\ CERN - Geneva \\ and \\ 0 . Pène \\ LPTHE, Orsay, France
}

\begin{abstract}
The pseudoscalar meson decay constant and the B parameter of charmed mesons are calculated by Monte Carlo simulation of lattice QCD, with Wilson fermions and in the quenched approxination. The combined results on two lattices, $10^{2} \times 20 \times 40$ and $16^{3} \times 48$, at $\beta=6$ and $\beta=6.2$ respectively, yield: $\mathrm{f}_{\mathrm{D}}=194 \pm 15$ and $\mathrm{f}_{\mathrm{F}}=215 \pm 17$, and $\mathrm{B}_{\mathrm{DD}}=0.82 \pm 0.10$ at $\mu=3 \mathrm{GeV}$. Within the non-relativistic quark model, predictions for the $b$ system can be obtained by extrapolation of the previous results: $\mathrm{f}_{\mathrm{B}_{\mathrm{d}}} \simeq 120 \mathrm{MeV}, \mathrm{f}_{\mathrm{B}_{\mathrm{s}}} \simeq 150 \mathrm{MeV}$. We have also computed the hyperfine splitting and photon coupling of the charmonium system: $M\left(1^{-}\right)-M\left(0^{-}\right)=50 \pm 10 \mathrm{MeV}$ (expt. $\left.117 \mathrm{MeV}\right),\left(\mathrm{f}_{\mathrm{J} / \psi}\right)^{-1} \simeq 0.100(\operatorname{expt}$. $0.125)$.
\end{abstract}

*) On leave of absence from LPTHE, Orsay, France. 
Charm was the first hadronic quantum number whose existence and appropriate mass were theoretically predicted before experimental observation. Charm and beauty decays deal with QCD at the interface between its perturbative and non-perturbative regimes. In particular, the study of open flavour states $\overline{Q q}$ constitutes a bridge between the theoretically quite well-understood heavy quarkonia $Q \bar{Q}$ spectra and the poorly-understood light $\bar{q} \bar{q}$ physics. Lattice QCD allows at present a rigorous treatment of the $q \bar{q}$ and $q \vec{Q}$ systems, at least for $Q=$ charm. This is important as, for instance, the extraction of the Kobayashi-Maskawa parameters in the standard model, which describes quark couplings, demands the understanding of hadronic couplings and bound states, which are the only ones experimentally accessible. obviously our chance of discovering any eventual new physics in hadronic decays is inversely proportional to our knowledge of these hadronic effects.

A serious step in this direction is the accurate determination of the pseudoscalar decay constants, such as $f_{D} \cdot f_{D}^{2}$ is a direct measure of the overlap of the heavy and light quarks in the $D$ meson, that is, of $|\psi(0,0)|^{2}$, the probability of the charm and light quark being at the same point. The rôle of $f_{D}$ in understanding charm decays is exemplified by the ratio of lifetimes,

$$
\frac{\tau\left(D^{+}\right)}{\tau\left(D^{0}\right)} \sim 2.5
$$

The departure from 1 of this ratio is a signal of the importance of hadronic effects. This means that the spectator model is not a good approximation, and annihilation and interference contributions proportional to $f_{D}$ are at work. Similar conclusions follow from the analysis of exclusive decays of the D mesons [1].

On the experimental side, the only information available is the Mark III upper bound [2]

$$
f_{D}<290 \mathrm{MeV} \quad(90 \% \text { C.L. })
$$

from the non-observation of $D^{+} \rightarrow \mu^{+} v$.

Another quantity besides the meson decay constants, essential for the understanding of weak heavy flavour transitions, is the so-called $B$ parameter. The $B$ parameter measures the ratio of the $(\mathrm{V}-\mathrm{A})(\mathrm{V}-\mathrm{A})$ matrix element (for instance, in the case of charm):

$$
B_{D^{0} \bar{D}}=\left\langle D^{0}\left|\bar{C} \gamma_{\mu}\left(1-\gamma_{5}\right) u \bar{C} \gamma^{\mu}\left(1-\gamma_{5}\right) u\right| \bar{D}^{0}\right\rangle
$$

to its vacuum insertion value $(8 / 3) \mathrm{m}_{\mathrm{D}}^{2} \mathrm{f}_{\mathrm{D}}^{2}$ (with the analogous $\mathrm{f}$ for the pion being $\left.f_{\pi}=132 \mathrm{MeV}\right)$. Again, an explicit lattice calculation of $B_{D^{0}} \vec{D}^{0}$ allows a comparison of the vacuum insertion approximation for light and heavy systems. 
The relevance of the parameters discussed above can be exemplified in the beauty sector. Very recent experimental results on $B^{0}-\vec{B}^{0}$ mixing [3], semileptonic branching ratios for $B$ mesons [4] and CP violation [5] are narrowing the bounds on the parameters of the $S U(2) \times U(1)$ model. Let us take the formula relating the top quark mass and $\mathrm{B}_{\mathrm{d}}-\overline{\mathrm{B}_{\mathrm{d}}}$ mixing $[6]^{*}$ ):

$$
\frac{r_{d}}{1-r_{d}}=\left(\frac{m_{t}}{10.8}\right)^{4} \frac{B_{B \bar{B}}^{2} f_{B d}^{4}}{(0.11 \mathrm{GeV})^{4}}\left(\frac{\tau_{b}}{1.1110^{-12} \mathrm{sec}}\right)^{2} \frac{1}{\left(s_{2}\right)^{4}}
$$

At fixed $r_{d}=r_{d}$ experimental, Eq. (2) shows the sensitivity of the top quark mass (and/or $s_{2}$ ) with respect to the theoretical prediction for $B_{B B}^{\frac{1}{2}} f_{B_{d}} \cdot s_{2}$ is the Kobayashi-Maskawa mixing angle $\left(v_{t d}=s_{l} \cdot s_{2}\right)$ and

$$
r_{\alpha}=\frac{\sigma\left(\bar{B}_{\alpha} B_{\alpha} \rightarrow \mu^{+} \mu^{+}\right)+\sigma\left(B_{d} \bar{B}_{\alpha} \rightarrow \mu^{-} \mu^{-}\right)}{\sigma\left(B_{\alpha} \bar{B}_{\alpha} \rightarrow \mu^{+} \mu^{-}\right)}
$$

for which the ARGUS result is $0.20 \pm 0.1 \mathrm{l}$. (QCD and higher $\mathrm{m}_{\mathrm{t}}^{2} / \mathrm{M}_{\mathrm{W}}^{2}$ corrections have been omitted for illustration purposes.) A similar analysis can be carried out for the $B_{s}-\overline{B_{s}}$ system, resulting in a refined analysis of the $C P$ violation effects in the kaon system [7].

As will be discussed later, technical 1 imitations forbid at present the propagation on the lattice of a bottom quark with a realistic mass. Any physical conclusion about the bottom system is just an extrapolation of charm results. on the contrary, we do have heavy quarks propagating with masses up to $\sim 1.2 \mathrm{GeV}$ $\left(\left(m_{c} a\right)^{2}<1\right)$, that is to say, the charm region.

It is plausible that, going from charm to beauty, a non-relativistic analysis of $f_{D}$ should make sense. In this case, $f_{D / B}$ are expected to behave as

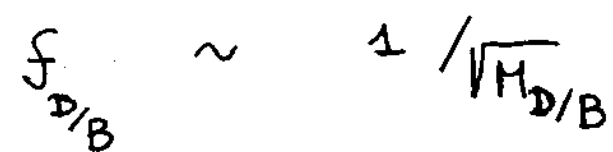

where $M$ is the meson mass. This behaviour should be corrected by a logarithmic

*) It should be stressed that the local approximation performed to obtain the effective $\Delta C=2(\Delta B=2)$ operators in the $B$ parameters is expected to be more accurate than the analogous procedure for the $K^{0}-K^{\theta}$ systems, as the masses involved are heavier $\left(m_{t}, m_{c}, m_{b}, \ldots\right)$. 
factor depending on $M$ due to the running of the QCD coupling constant when comparing different scales [8]. In the same approximation, the B-parameter is expected to be approximately constant.

An alternative approach has recently been proposed [9] to study $\vec{Q}$ systems by a systematic expansion in $1 / M_{Q}$. Finally, we recall that results for $f_{D / B}$ and the $B$-parameter have been obtained with QCD sum rules. We compare the latter results with ours at the end of this paper.

A lattice Monte Carlo evaluation of the pseudoscalar decay constants $f_{\pi}$, $f_{K}$, $f_{D}, \ldots$ implies the analysis of two-point correlation functions on Euclidean space-time. We will consider the following vacuum expectation values:

$$
\begin{aligned}
& G_{A_{0} P_{5}}(t)=\sum_{\vec{x}}\left\langle 0\left|A_{0}(\vec{x}, t) P_{5}^{+}(0,0)\right| 0\right\rangle \simeq \overrightarrow{t a\rangle}_{a} \frac{\left\langle 0\left|A_{0}\right| M\right\rangle\left\langle H\left|P_{5}^{+}\right| 0\right\rangle e^{-m m_{H}}}{2 m_{M}} \\
& G_{5}(t)=\sum_{\vec{x}}\left\langle 0\left|P_{5}(\vec{x}, t) P_{5}^{+}(0,0)\right| 0\right\rangle \simeq \underset{t a\rangle 1}{\simeq} \frac{\left\langle 0\left|P_{5}\right| M\right\rangle\left\langle M\left|P_{5}\right| 0\right\rangle e^{-m M_{M} t}}{2 m_{M}}
\end{aligned}
$$

In these expressions $M$ is the lowest intermediate state for a given pseudoscalar source $P_{5}(x)=\bar{\psi}(x) \gamma_{5} \psi$ on the lattice, with relative normalization constant $Z_{5}$

$$
Z_{5}^{1 / 2}=\left\langle 0\left|P_{5}^{+}\right| M\right\rangle
$$

$A_{0}$ is the fourth component of the axial current defined on the lattice,

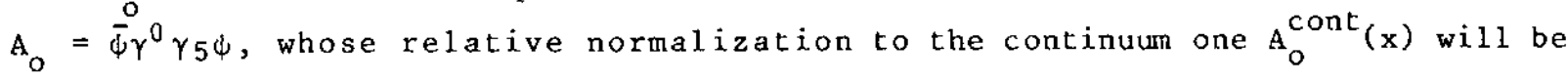
denoted by $z_{A}$

$$
\left\langle\alpha\left|A_{0}\right| \beta\right\rangle=z_{A}^{-1}\left\langle\alpha\left|A_{0}^{\text {cont }}\right| \beta\right\rangle
$$

The ratio of $G_{A_{0}} P_{5}(t)$ to $G_{5}(t)$ for large $t$ gives the desired physical information 


$$
\begin{aligned}
R_{M}(t)= & \frac{\sum_{\bar{x}}\left\langle 0\left|A_{0}(\bar{x}, t) P_{5}^{+}(0,0)\right| 0\right\rangle}{\sum_{\bar{x}}\left\langle 0\left|P_{5}(\bar{x}, t) P_{5}^{+}(0,0)\right| 0\right\rangle} \simeq \underline{t a\rangle 1} \frac{\left\langle 0\left|A_{0}\right| M\right\rangle}{\left\langle 0\left|P_{5}\right| M\right\rangle} \\
& =\frac{f_{M} m_{M}}{Z_{A} Z_{5}^{1 / 2}}
\end{aligned}
$$

$Z_{5}$ can be extracted from a fit of the pseudoscalar-pseudoscalar correlation function, whose exponential decay should be dominated by the lowest-lying meson state for large enough lattice distances, as assumed in Eqs. (4) and (5).

The evaluation of $\mathrm{Z}_{\mathrm{A}}$ can be done in principle in perturbation theory [10]. In Ref. [11], the normalization factor of the extended axial current, $\mathrm{Z}_{A_{e x}}$, was computed non-perturbatively from current algebra relations. The value of $\mathrm{Z}_{\mathrm{A}}$ found in Ref. [11] differs from the perturbative result [12] by about $10-15 \%$ in the range of lattice coupling constants considered here. Since this non-perturbative calculation has not been done for the local axial current, we will use the perturbative value of $Z_{A}$ throughout this paper. One has to bear in mind that a $10-15 \%$ error is expected also in this case.

Equation (8) is subject to systematic effects, the most obvious one being the dependence on the lattice spacing a. But this dependence is factorizable and can be avoided by considering ratios of pseudoscalar decay constants, and normalizing the results to $\mathrm{f}_{\pi}$. This is equivalent to fixing the lattice spacing, a, by setting $f_{\pi}$ to its physical value. Considering the dimensionless quantity $R$, Eq. (8), for D and $\pi$ mesons, we can take the ratio

$$
\frac{R_{D}(t)}{R_{\pi}(t)} \underset{t a \gg 1}{\simeq} \frac{f_{D} m_{D}}{f_{\pi} m_{\pi}}\left[\frac{z_{5}\left(m_{\pi}\right)}{z_{5}\left(m_{D}\right)}\right]^{1 / 2}
$$

In the equation above, $Z_{A}$ cancels, since it is independent of the quark mass $m_{q}$ as far as $m_{q} a \ll 1$. We have explicitly verified that the renormalization constant of the local vector current $\left(\bar{\psi}_{\gamma}^{\mu} \psi\right) z_{v}$ is indeed practically independent of the quark mass in the range of masses ("charm" included) considered in this paper (see below). This will certainly not be true whenever $m_{q} a \simeq 1$.

For the vector meson decay constants, the relevant two-point Green function is: 
$-5-$

$$
\begin{gathered}
\sum_{\bar{x}}\left\langle 0\left|V(\bar{x}, t) V_{k}(0,0)\right| 0\right\rangle \simeq \frac{\tilde{t a}\rangle\rangle \mid}{\left\langle 0\left|V_{k}\right| V\right\rangle\left\langle V\left|V_{k}\right| 0\right\rangle} e^{-m_{V} t} \\
\frac{2 m_{V}}{}
\end{gathered}
$$

where $V_{\mu}$ is the lattice vector current

$$
\left\langle\alpha\left|V_{\mu} \quad\right| \beta\right\rangle=z_{V}^{-1}\left\langle\alpha\left|V_{\mu}^{\text {cont }}\right| \beta\right\rangle
$$

and

$$
\left\langle 0\left|V_{k}\right| V\right\rangle=\frac{1}{f_{V}} m_{V}^{2} \epsilon_{k}
$$

For the B parameter, the evaluation of three-point functions is necessary

$$
\begin{aligned}
& G_{i}\left(t_{1}, t_{2}\right)=\sum_{\bar{x}, \bar{y}}\left\langle 0\left|T\left(P_{5}\left(\bar{x}, t_{1}\right) O_{i}^{\text {LAT }}(0) P_{5}\left(\bar{y}, t_{2}\right)\right)\right| 0\right\rangle \\
& \simeq \quad \frac{\left\langle 0\left|P_{5}\right| M\right\rangle\left\langle M\left|O_{i}^{(A T T}(0)\right| \bar{M}\right\rangle\left\langle\bar{M}\left|P_{5}\right| 0\right\rangle}{\left(2 m_{M}\right)^{2}} \\
& \left.\left|t_{1}\right| a\right\rangle>1 \\
& \left(2 m_{M}\right)^{2} \\
& \left.\left.\left|t_{2}\right| a\right\rangle\right\rangle \mid \\
& \times e^{-m_{M}\left(|t,|+\left|t_{2}\right|\right)}
\end{aligned}
$$

with $-\mathrm{T} / 2<\mathrm{t}_{1}<0<\mathrm{t}_{2}<\mathrm{T} / 2$, where $\mathrm{T}$ is the period of the lattice in the time direction.

The relation between the lattice operators $o_{i}^{\text {LATI }}$ and the continuum ones

$$
O_{\Delta C=2} \equiv \bar{c} \gamma_{\mu}\left(1-\gamma_{5}\right) u-\gamma^{\mu}\left(1-\gamma_{5}\right) u
$$

in a given renormalization prescription is

$$
O_{\Delta C=2}=Z_{L A T T}\left(\mu a, g_{0}^{(a))}\left[O_{\Delta C=2}^{L A T T}+\sum_{i} \delta_{6}^{i} O_{i}^{L A T T}\right]\right.
$$

where $\mu$ is the renormalization point, $g_{0}$ the lattice coupling, and $O_{\triangle \mathrm{C}=2}^{\mathrm{LATT}}$ is the naive lattice bare operator formally equal to Eq. (14). The sum on $i$ runs over dimension- six operators of different chiralities [13] such as $(\bar{c} d)^{2},\left(\bar{c} \gamma_{5} d\right)^{2}, \ldots$. 
Note that there are no lower dimension operators for $\Delta \mathrm{C}=2$, in contrast with $\Delta C=1$ transitions. A perturbative computation of the Wilson coefficients, $\delta_{6}^{i}$ and $\mathrm{Z}_{\text {LATT }}$, can be considered a reasonable approximation. In fact, the results obtained for soft pion theorems [14] in $\Delta I=3 / 2$ kaon transitions show that these perturbative subtractions are reliable.

The physical matrix element is thus extracted:

$$
\begin{aligned}
& B\left(t_{1}, t_{2}\right)=Z_{A}^{-2} \sum_{\bar{x}, \bar{y}}\left\langle 0\left|P_{5}\left(\bar{x}, t_{1}\right) O_{\Delta c=2}(0) P_{5}\left(\bar{y}, t_{2}\right)\right| 0\right\rangle \\
& \frac{8}{3}\left(\sum_{\bar{x}}\left\langle 0\left|A_{0}(\bar{x}, t) P_{5}^{t}(0,0)\right| 0\right\rangle\right)\left(\sum_{\bar{y}}\left\langle 0\left|A_{0}\left(\vec{y}, t_{2}\right) P_{5}^{t}(0,0)\right| 0\right\rangle\right) \\
& \frac{\left\langle D^{0}\left|O_{\Delta C}=2\right| \bar{D}^{0}\right\rangle}{\frac{8}{3} f_{D}^{2} M_{D}^{2}}
\end{aligned}
$$

which for $a\left|t_{1}\right|, a\left|t_{2}\right| \gg 1$ is just the $B$ parameter for charm.

We now describe our numerical analysis, which uses wilson fermions in the quenched approximation. The results of this paper have been obtained from two different Monte Car1o studies. The first was performed on a $20 \times 10 \times 10 \times 40$ lattice at $\beta=6$. We generated 15 independent gauge field configurations, in the quenched approximation, on a $10^{3} \times 20$ lattice which was subsequently duplicated in the $x$ and $t$ directions. We estimate that the systematic error involved in the duplication procedure is much smaller than other statistical systematic errors present in the computation of the various quantities of interest. For any of the configurations, we have computed the quark propagator at three values of the Wilson hopping parameter, $K=0.1515,0.1530$ and 0.1545 , and at the value $K=0.1350$ corresponding to the "charm" quark. A second Monte Carlo simulation was done on a $16^{3} \times 48$ lattice at $\beta=6.2$ (without any duplication of the gauge field configurations). We generated 15 independent gauge field configurations and evaluated the quark propagator at $K=0.1500,0.1510$ and 0.1520 , and $K=0.1350$ for "charm". The volume was scaled to match approximately the physical size of the two lattices in Fermi units $\left[a^{-1}(\beta=6.2) /\left(a^{-1}(\beta=6) \simeq 1.6\right]\right.$.

The analysis of the spectrum for light particles [14] translates these parameters into the physical ones. If we determine $a^{-1}$ from the $\rho$ mass at critical $k$, we find:

$$
\left.\begin{array}{l}
\left.a^{-1}=2.2 \pm 0.10 \mathrm{GeV} \text { at } \beta=6.0\right\} \\
\left.a^{-1}=3.0 \pm 0.20 \mathrm{GeV} \text { at } \beta=6.2\right\}
\end{array}\right\} \text { method I }
$$


with the lightest pion weighing about $650 \mathrm{MeV}(650 \mathrm{MeV})$ and a charmed quark mass $\left.{ }^{*}\right)$ of about $1.0 \mathrm{GeV}(1.3 \mathrm{GeV})$ at $\beta=6.0(\beta=6.2)$. Alternatively, one can determine $a^{-1}$ from the slope of the vector meson spectrum versus the pseudoscalar mass squared $[11,14]$. In this case, one finds

$$
\begin{aligned}
& \left.a^{-1}=1.6 \pm 0.10 \mathrm{GeV} \text { at } \beta=6.0\right\} \\
& \left.a^{-1}=2.2 \pm 0.20 \mathrm{GeV} \text { at } \beta=6.2\right\}
\end{aligned}
$$

A systematic error of about $\pm 400 \mathrm{MeV}$ is implied. A result similar to method II is obtained by determining $a^{-1}$ from $f_{\pi}$. In the following, we will scale $f_{D, F}$ with $f_{\pi}$ (i.e., use method II) and give mass differences in units of the $\mathrm{J} / \Psi$ mass.

To estimate the statistical errors, we have divided our 15 configurations on each lattice into three clusters of five, and measured the relevant correlation functions over each cluster. For any quantity $x$, we compute the best value $\bar{x}$ and three values $x_{c l u}$, obtained from the correlation functions mediated over the whole set of configurations or over each cluster respectively. The reported value of $X$ is then $X=\bar{X}+\Delta X$

$$
\Delta x^{2}=\frac{1}{2}\left[\frac{1}{3} \sum_{\text {cluster }} X_{\text {clust. }}^{2}-\left(\frac{1}{3} \sum_{\text {censter }} X_{\text {clust. }}\right)^{2}\right]
$$

Whenever the results from the two lattices are combined, errors will be added in quadrature, unless indicated otherwise.

The results for the charmed meson spectrum and the charmonium system from the Paris $\left(16^{3} \times 48\right)$ lattice are given in Table 1. In this Table, whenever u and d quarks are considered, we have extrapolated to the critical kappa, $\mathrm{K}_{\mathrm{c}}$, corresponding to the chiral 1 imit $\mathrm{m}_{\mathrm{q}}=0$. For reference, we give here the value of the $\mathrm{J} / \phi$ mass:

$$
\begin{aligned}
& M_{J / \Psi}=(3.25 \pm 0.02) \mathrm{GVV} \quad \text { method I } \\
& M_{J / \Psi}=(2.38 \pm 0.01) \mathrm{GeV} \quad \text { method II }
\end{aligned}
$$

The above values, as well as the values in Table 1, are from the Paris lattice, $\beta=$ 6.2 , for which we have a realistic charmed quark mass. Comparing the $\eta_{c}$ and $J / \psi$

*) All the quark masses above are defined as $m_{g}=\frac{1}{2}\left(1 / \mathrm{k}-1 / \mathrm{k}_{\mathrm{c}}\right)$. All the renormalization factors and the renormalization necessary to relate the lattice quark mass to the continum one have been omitted. For a detailed discussion, see Ref. [11]. 
masses, the resulting hyperfine splitting is $50 \pm 10 \mathrm{MeV}$, to be compared with the experimental value $117 \mathrm{MeV}$. This discrepancy can be associated to the systematic errors which appear for heavy quark systems, namely the fact that when $m$ grows, corrections of order (ma) can be important (for us ma ${ }_{\text {charm }} \simeq 0.4$ ), as wel1 as to the effect of quenching. In general, while statistical errors for heavy systems are smaller than for light ones, systematic errors of the type just mentioned are bigger.

We have estimated the value of $Z_{V}$ from two-point correlation functions of vector currents, following Ref. [11], for charmed quarks and found that its variation with respect to the light quark is tiny, $Z_{V}(1$ ight $)=0.62, z_{v}$ (heavy) $=0.64$. This is to be expected, as $\mathrm{Z}_{\mathrm{V}}$ should depend on $\mathrm{g}_{\mathrm{o}}$ only if the masses involved can be neglected with respect to the renormalization point $z=z\left(g_{0}\right)\left(1+o\left(m^{2} a^{2}\right)\right)$, which is still the case for our charmed quark. We have not explicitly calculated the three-point functions necessary for the determination of $\mathrm{Z}_{\mathrm{A}}$ (heavy). Note, however, that in this context the ratios of pseudoscalar meson constants are independent of $\mathrm{Z}_{\mathrm{A}}$, on contrast to the $\mathrm{B}$ parameter, Eqs. (9) and (16).

In Table 2, we present the results for the meson decay constants normalized to $f_{\pi}\left(f_{\pi}^{e x p}=132 \mathrm{MeV}\right)$, and for the $B$ parameter for charmed hadrons. In Fig. 1 , the ratio in Eq. (16) is reported and seen to be quite constant for time distances large enough. If we combine the results at $\beta=6$ and $\beta=6.2$ (neglecting a possible charm mass dependence), we obtain

$$
\mathrm{B}_{\mathrm{D} \overline{\mathrm{D}}}=0.82 \pm 0.10 \text { at } 3 \mathrm{GeV}
$$

For comparison, we recall the result for the kaon system [14],

$$
\mathrm{B}_{\mathrm{K} \overline{\mathrm{K}}}=0.65 \pm 0.15
$$

at the same scale. With four flavours, and $\Lambda_{Q C D}=200 \mathrm{MeV}$, the renormalization group invariant $B$ parameter is:

$$
\begin{aligned}
B_{D \bar{D}} & =B_{D \bar{D}}(\mu=3 \mathrm{GeV})\left[\alpha_{s}(\mu)\right]^{-6 / 25} \\
& =1.11 \pm 0.14
\end{aligned}
$$




\section{CONCLUSIONS}

Our previous results [14] on the pseudoscalar decay constant showed good agreement with experiment for light systems. The present results for $f_{D}$ and $f_{F}$ (Table 2) can be compared with QCD sum rule predictions. Although sevexal sum rule analyses [15] predict higher values than ours, such as $f_{D}=226 \pm 21 \mathrm{MeV}, f_{F}=$ $274 \pm 17 \mathrm{MeV}$, our results are consistent with both early sum rule estimates [16] ( $f_{D}$ $\left.=226 \pm 21, \mathrm{f}_{\mathrm{F}}=274 \pm 17 \mathrm{MeV}\right)$ and very recent refined ones [17] $\left(\mathrm{f}_{\mathrm{D}}=(172 \pm 15) \mathrm{MeV}\right.$, $\left.f_{F} \simeq 220 \mathrm{MeV}\right)$. Note that, as explained above, a11 f's have been estimated computing ratios $f / f \pi$ and are, in consequence, almost free of the systematic errors associated with the lattice spacing a.

The combined results for the $B_{D D}$ parameter in the charmed mass region are bigger than the corresponding ones for the kaon system, $B_{K \bar{K}}=0.65 \pm 0.15$, at the same scale of $\sim 3 \mathrm{GeV}$. Within large errors, this result seems to support the idea that, at the same renormalization scale, the B parameter is closer to one for heavy quark systems.

The extrapolation to the $B$ mesons reported in Table 2 is based on the nonrelativistic quark model picture. When the heavy quark mass grows, $m_{Q} \rightarrow \infty$, compared to $\Lambda_{Q C D}, Q$ is a fixed force centre, while the light quark is smeared around over a distance $1 / R$, the confinement radius. This implies a scaling behaviour for $\mathrm{f}_{M}, \mathrm{f}_{M} \sim \mathrm{M}_{\mathrm{Q}}^{-\frac{1}{2}}$. It has been pointed out [8] that in the non-relativistic approach, $\mathrm{f}_{\mathrm{M}}$ is defined at $\mu \sim \mathrm{R}^{-1}$, while the axial current corresponding to the definition of the physical $\mathrm{f}_{M}, \mathrm{f}_{\mathrm{M}}=\left\langle 0\left|\bar{\psi}_{\gamma_{\mu}} \gamma_{5} \psi\right| \mathrm{P}\right\rangle$, is renormalized at a higher scale $\mu \sim M_{Q}$, and the running of the QCD coupling constant will modify the extrapolation law. However, this correction is of the order of $10 \%$ and, given our error bars, we have left it out. The main objection to this extrapolation comes, in our opinion, from the fact that we do not know if the charm mass is large enough for the asymptotic law to start to be valid. Our prediction for $f_{B_{d}} \sim 120$ MeV can be compared with recent [17] sum rule analyses $\left.\left(f_{B}=187 \pm 17\right) \mathrm{MeV}\right)$, although again a wider range of values appeared in previous sum rule literature $[15,16]$. What seems to be a common trend to these predictions is $\mathrm{f}_{\mathrm{B}_{\mathrm{d}}} / \mathrm{f}_{\pi}>1$, contrary to our extrapolation.

An illustrative application of our extrapolations for the beauty system is obtained by translating our results in terms of the $B_{B_{d}}{ }^{-B_{B}}$ mixing and the top mass mentioned above. Given the experimental error bars as well as ours, we cannot set an improved rigorous $1 \mathrm{imit}$ on $\mathrm{m}_{t}$. Should we consider our central values as an indication, our data favour rather high values for $\mathrm{m}_{t}$. For instance, assuming $\mathrm{B}_{\mathrm{B} \overline{\mathrm{B}}} \simeq 1, \mathrm{f}_{\mathrm{B}_{\mathrm{d}}}$ from Table 2 would favour $\mathrm{m}_{t} \geqslant 100 \mathrm{GeV}$, following the analysis of Ref. [18] with $\tau_{D}=1.11 \cdot 10^{17} \mathrm{sec}$. Lower values of $\mathrm{m}_{\mathrm{t}}$ are not excluded as our 
error bars are of the order of $30 \%$ and an extrapolation is involved. But we are confident in the general trend.

Another interesting consequence stems from the theoretical analysis of the CP violation parameter $\varepsilon$ [7]. The dependence of $\varepsilon$ on $\mathrm{m}_{t}$ in the standard model includes a term logarithmic in $m_{t}$ and a term quadratic. For 1 arge $m_{t}$, the 1 ast one dominates to a good approximation, and using Eq. [3] for the dependence on $s_{2}$ and $\mathrm{m}_{t}$, the result is

$$
\epsilon \sim 0.30 s_{3} s_{\alpha} \sqrt{1-\left(\frac{s_{3} s_{\alpha}}{0.05}\right)^{2}} \frac{f_{k}^{2} B_{k K}}{\frac{f_{B_{\alpha}}^{2} B_{B_{\alpha}} \bar{B}_{\alpha}}{S^{2}}}
$$

Considering our central values in Eqs. (18) and (19), this result would translate into $s_{3} s_{d}<0.5 \cdot 10^{-2}$, and $s_{3} s_{d}<0.7 \cdot 10^{-2}$ if one includes also the logarithmic corrections, with an error not bigger than $40 \%$. Again this is just an amusing indication as long as an accurate lattice determination of $\mathrm{f}_{\mathrm{B}_{\mathrm{d}}}^{2}{ }_{\mathrm{B}_{\mathrm{d}}} \overline{\mathrm{B}_{\mathrm{d}}}$ is not available.

Most of the results contained in this note (in a preliminary version) have been previously reported at the 1987 EPS Conference [19] and at the Seillac Workshop [20]. Our results for $\mathrm{f}_{D, F}$ are in agreement within the errors with those of Ref. [21].

\section{ACKNOWLEDGEMENTS}

We are indebted to A. Pich for many useful discussions. For their invaluable help we thank the members of the CcVR assistance of the Ecole Polytechnique and of the CINECA in Bologna. The support from CNRS and INFN is gratefully acknowledged. These computations were done using the CRAY-XMP48 of CINECA and the CRAY-2 of the Ecole Polytechnique. 


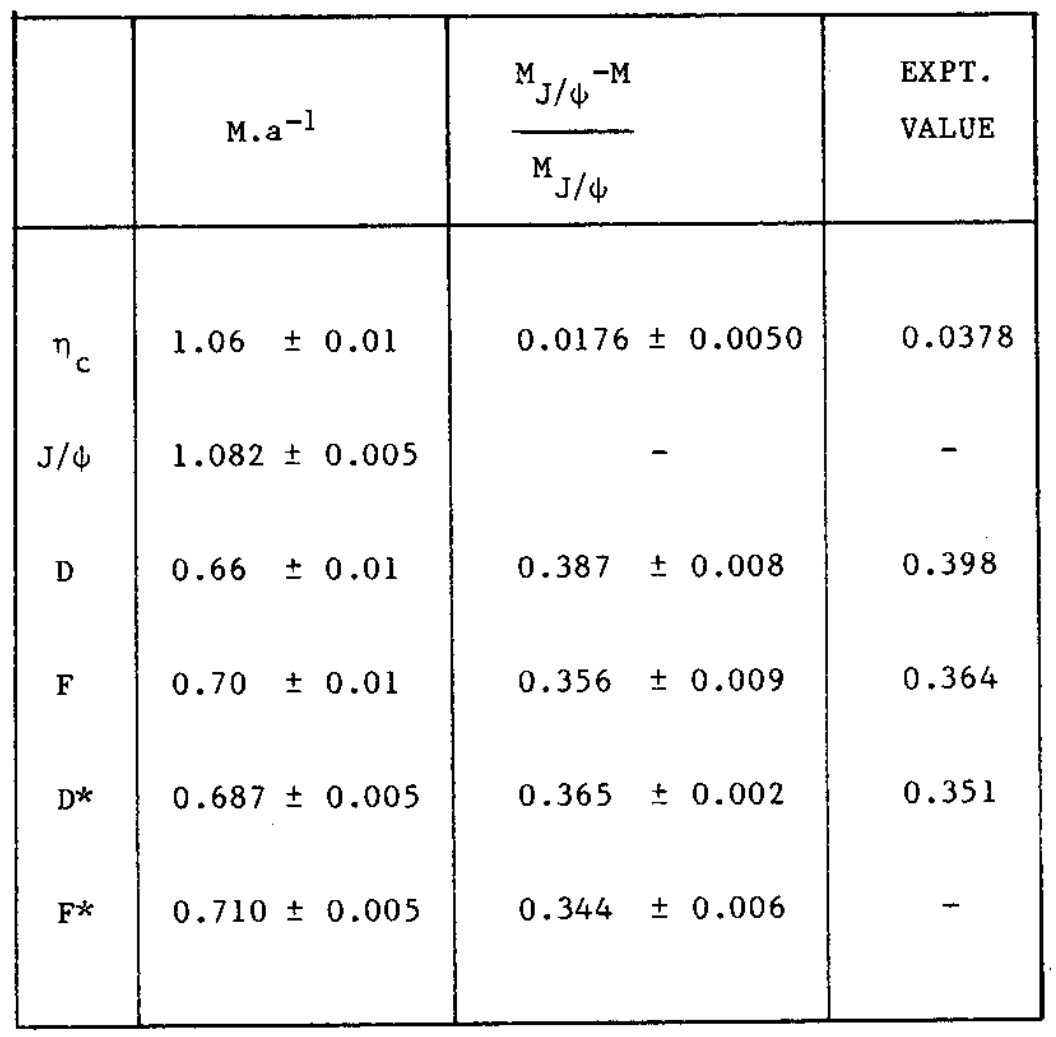

TABLE 1

Charmed mesons and charmonium spectrum from the $\beta=6.2$ lattice.

First column : masses in lattice units.

Second column: mass differences relative to the $\mathrm{J} / \psi$.

Third column : experimental values. 


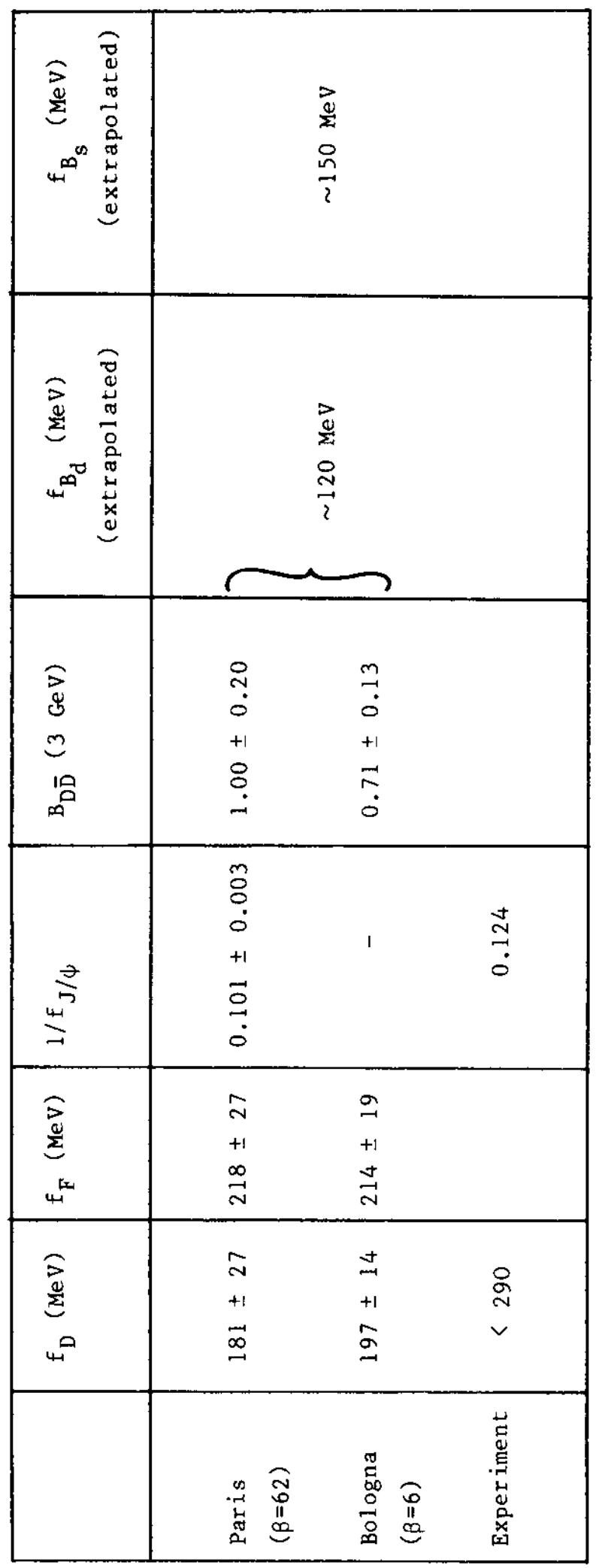

离

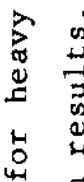

空焉

$m \stackrel{\text { ल }}{-}$

II

$z 0$ in

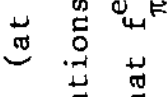

\&

점

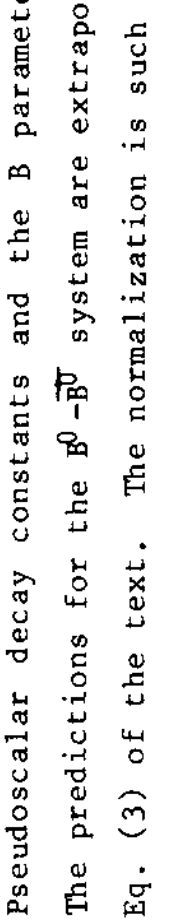




\section{REFERENCES}

[1] I.I. Bigi, SLAC-PUB 4455 (1987).

[2] J. Adler et al., SLAC-PUB-4343 (1987).

[3] UAl Collaboration, C. Albajar et al., Phys. Lett. 186B (1987) 247.

[4] ARGUS Collaboration, H. Schröder, XXII Rencontre de Moriond (Les Arcs, France, March 1987).

[5] F.H. Thorndike, in Proceedings of the 1985 Int. Symposium on Lepton and Photon Interactions at High Energies, eds. M. Konuma and K. Takahashi, Kyoto 1985.

[6] J.F. Donoghue et al., Phys. Lett. 195B (1987) 295.

[7] J.F. Donoghue et al., Phys. Lett. 195B (1987) 285.

[8] I. Shifman and M. Voloshin, ITEP preprint 86-54 (1986);

I. Shifman, Proceedings of the Inst. Symposium on Production and Decay of Heavy Flavours, Heidelberg, May 1986.

[9] E. Eichten, Talk delivered at the "International Symposium of Field Theory on the Lattice", Seillac, France, 2 September - 2 October 1987.

[10] M. Bochicchio et al., Nuc1. Phys. B262 (1985) 331 ; see also:

G. Curci, Phys. Lett. 167B (1986) 425.

[11] L. Maiani and G. Martinel1i, Phys. Lett. 178B (1986) 265.

[12] B. Meyer and C. Smith, Phys. Lett. 123B (1983) 62;

G. Martinelli and Y.C. Zhang, Phys. Lett. 123B (1983) 77;

G. Martinel1i and Z.Y. Cheng, Phys. Lett. 125B (1983) 7;

R. Groot, J. Hoek and J. Smit, Nuc1. Phys. B237 (1984) 11.

[13] G. Martine11i, Phys. Lett. 141B (1984) 395;

C. Bernard et a1., UCLA/87/TEP/17 (May 1987).

[14] M.B. Gavela et al., CERN-TH.4905/87 (November 1987).

[15] See, for instance:

C.A. Dominguez and N. Pava, Phys, Lett. 197B (1987) 423, and references therein.

[16] S.C. Generalis, Ph.D. thesis, Open University Report No. OUT-4102-13 (1984); A.R. Zhitnitskii, I.R. Zhitnitskii and V.L. Chernya, Sov. J. Nuc1. phys. 38 (1983) 775;

T.M. Al iev and V.L. Eletsky, Sov. J. Nuc1. Phys. 38 (1983) 936.

[17] S. Narison, CERN-TH.4768/87, PM 87/33 (1987).

[18] J. Ellis, J.S. Hagelin and S. Rudaz, Phys. Lett. 192B (1987) 201;

J. Maalampi and M. Roos, Phys. Lett. 195B (1987) 489. 
[19] 0. Pene, Invited talk at the EPS-HEP Conference (Uppsala), June 1987, to appear in the proceedings.

[20] M.B. Gavela et al., talk presented by L. Maiani at the "International Symposium of Field Theory on the Lattice", Seillac, France, 28 September 2 october 1987.

[21] T.A. DeGrand and R.D. Loft, preprint CoLO-HEP-165 (November 1987).

\section{FIGURE CAPTION}

$B\left(t_{1}, t_{2}\right)$ defined in Eq. (16) at $\beta=6.2$ with $K=0.1500$ is reported as a function of $t_{2}$ at fixed $t_{1}$. For $T / 2 \ll t_{2} \ll T$, the three-point correlation fucntion is dominated by the matrix element $\left\langle D^{0}\left|O_{\Delta C=2}\right| \bar{D}^{0}\right\rangle$ and is approximately equal to the right-hand side of Eq. (16). 


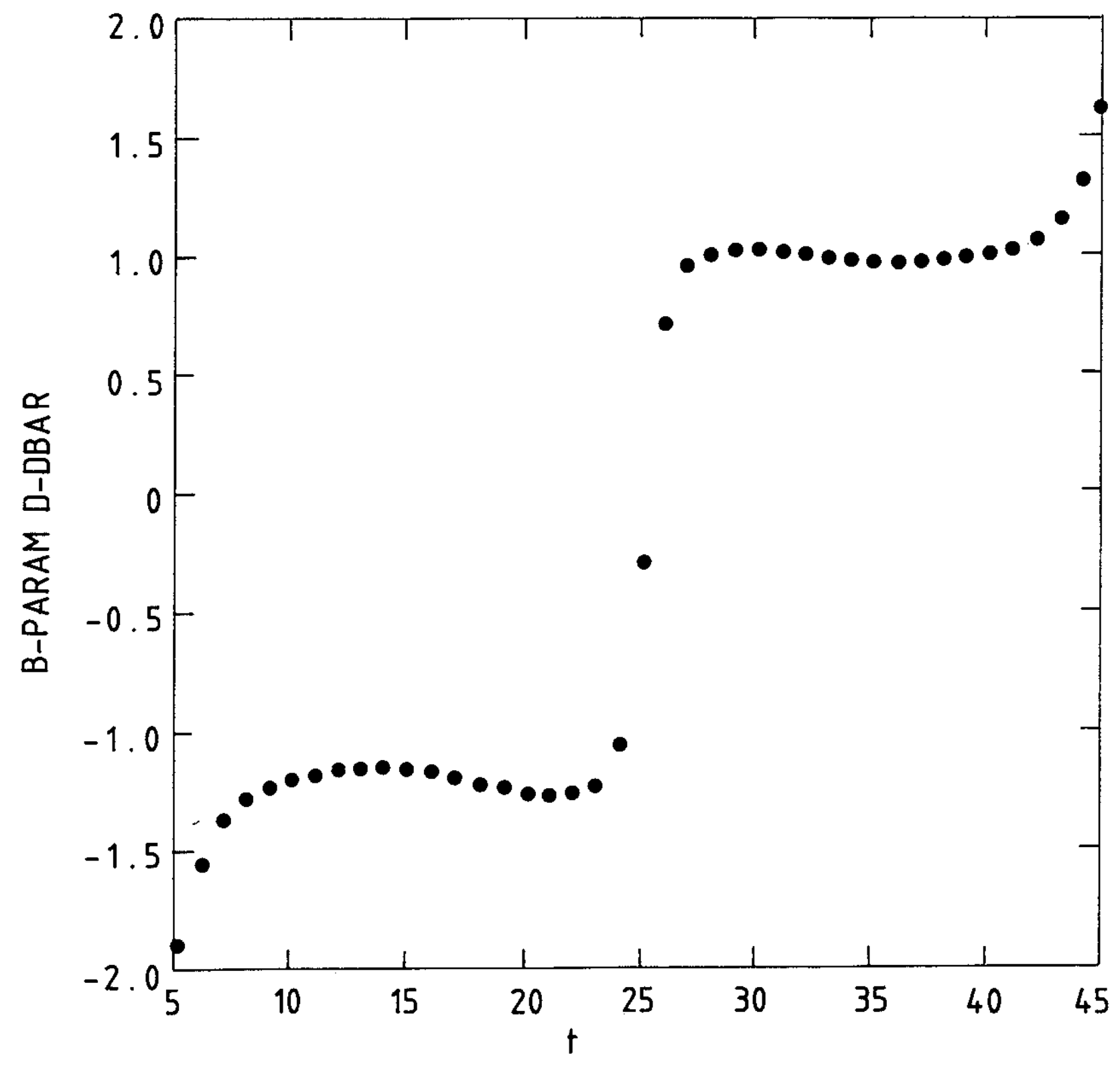

